\title{
Spectrum Harvesting and Sharing in Multi-Hop CRNs Under Uncertain Spectrum Supply
}

\author{
Miao Pan, Student Member, IEEE, Chi Zhang, Student Member, IEEE, Pan Li, Member, IEEE and \\ Yuguang Fang, Fellow, IEEE
}

\begin{abstract}
The essential impediment to apply cognitive radio (CR) technology for efficient spectrum utilization lies in the uncertainty of licensed spectrum supply. In this paper, we propose a novel architecture for spectrum harvesting and sharing, and investigate the joint routing and frequency scheduling problem in multi-hop cognitive radio networks (CRNs) under uncertain spectrum supply. We introduce a new service provider, Secondary Service Provider (SSP), to facilitate the accessing for secondary users (SUs). We model the vacancy of available bands with a series of random variables, and mathematically describe the corresponding frequency scheduling and flow routing constraints. From the SSP's point of view, we characterize the CRN performance with a pair of parameters $(\alpha, \beta)$, and present an optimization problem to minimize the required network-wide spectrum resource at the $(\alpha, \beta)$ level. Given that $(\alpha, \beta)$ level is specified, we obtain a lower bound for the optimization problem and develop a threshold based coarse-grained fixing algorithm for a feasible solution. Simulation results show that i) for any $(\alpha, \beta)$ level, the proposed algorithm provides a near-optimal solution to the formulated NP-hard problem, and ii) the $(\alpha, \beta)$ based solution is better than the expected bandwidth based one in terms of blocking ratio and spectrum utilization in multi-hop CRNs.
\end{abstract}

Index Terms-Cognitive radio networks; Spectrum harvesting; Spectrum allocation; Routing; Scheduling; Cross-layer optimization.

\section{INTRODUCTION}

$\mathbf{R}$ ECENT years have witnessed the boosting growth of wireless networks and flourish of various wireless services. In parallel with that, current static spectrum allocation policy of Federal Communications Commission (FCC) [2][4] results in the exhaustion of available spectrum, while a lot of licensed spectrum bands are extremely under-utilized. Experimental tests in academia [5], [6] and measurements conducted in industries [7], [8] both show that many licensed spectrum blocks are not used in certain geographical areas and are idle most of the time. Even in the most crowded

Manuscript received 15 February 2011; revised 20 July 2011. This work was supported by the National Science Foundation under grants CNS0721744, CNS-0916391, ECCS-1129062, and ECCS-1128768. The work of Y. Fang was also partially supported by the National Natural Science Foundation of China under grant 61003300 . The preliminary version has been presented at IEEE INFOCOM'2011 [1].

M. Pan and Y. Fang are with the Department of Electrical and Computer Engineering, University of Florida, Gainesville, FL 32611, USA. (e-mail: miaopan@ufl.edu; fang@ece.ufl.edu). Y. Fang is also a Changjiang Scholar Chair Professor with the National Key Laboratory of Integrated Services Networks, Xidian University, Xi'an, China

C. Zhang is with the School of Information Science and Technology, University of Science and Technology of China, Hefei 230027, China (email: chizhang@ustc.edu.cn).

P. $\mathrm{Li}$ is with the Department of Electrical and Computer Engineering, Mississippi State University, Mississippi State, MS 39762 USA (e-mail: li@ece.msstate.edu).

Digital Object Identifier 10.1109/JSAC.2012.120216. area near downtown Washington, DC, where both government and commercial spectrum use is intensive, only $38 \%$ of the licensed spectrum remains occupied and the rest of spectrum resource (a.k.a., "white space/spectrum hole") is wasted. These statistics and studies spur the FCC to open up licensed spectrum bands and pursue new innovative technologies to encourage dynamic use of the under-utilized spectrum [2].

As one of the most promising solutions, cognitive radio (CR) technology releases the spectrum from shackles of authorized licenses, and allows opportunistic usage of the vacant licensed spectrum bands in either temporal or spatial domain. Due to the potential of greatly improving the spectrum utilization, CR technology promotes numerous possible applications in various areas, e.g., military communications, public safety, disaster relief, search and rescue, environment monitoring, etc.

However, to opportunistically access the licensed bands, CR devices have to be frequency-agile [3], [9]. It is imperative for the $\mathrm{CR}$ devices to have the capability of exploring licensed spectrum bands, reconfiguring RF, switching frequencies across a wide spectrum range (i.e., from $20 \mathrm{MHz}$ to $2.5 \mathrm{GHz}$ [9], [10]), sending and receiving packets over non-contiguous spectrum bands, etc. Although some of the desired features may be realized in the future, enormous amount of time and efforts must be spent in hardware designs and signal processing in order to implement these features in light weight radios [3], [9], [10]. In addition, to attract customers for any new technologies, there is no reason to enforce the users to replace their communication devices or to increase the complexity on the customers' side. For cognitive radio networks (CRNs), it is always a good idea to minimize the changes on the handsets of secondary users (SUs) while maximizing the spectral efficiency. Thus, it may be more viable to design a new architecture of networks to effectively harvest white space while minimizing the complexity of SUs.

Another key obstacle to the employment of multi-hop CRNs lies in the uncertain licensed spectrum supply [3], [4], [11]. Since the CR services must evacuate the licensed bands when primary services are active, the returning of primary services has significant impact on how to perform opportunistic spectrum accessing (OSA), scheduling and interference avoidance, and multi-hop multi-path routing in CRNs. Stateof-the-art work on investigating the unpredictable activities of primary services can generally be classified into two categories: i) spectrum sensing and ii) statistical analysis of the collected/historical spectrum vacancy/occupancy data.

Specifically, in spite of the overwhelming waste of sensing time which can be used for more traffic delivery, individual sensing is trapped by sensing accuracy since both false alarm 
probability and missing detection probability are really high. To overcome the weakness of individual sensing, cooperative sensing is proposed to improve the sensing accuracy by grouping CR devices to sense together and share information among the group. But the trouble is that it is too difficult to synchronize the $\mathrm{CR}$ devices in the group to sense simultaneously. In addition, cooperative sensing has to set up a common channel for information exchange, which will incur enormous communication overhead. On the other hand, in [5], [7], [8], researchers as well as engineers try to identify the spectrum supply for OSA with the statistics of licensed spectrum utilization rather than attempting to detect the activities of primary services. They have carried out spectrum measurements, collected and analyzed the data about spectrum utilization, and summarized the statistical characteristics of the band vacancy/occupancy in details. These statistical results contain abundant information about the activities of primary services and provide a nice guide to the CR devices for OSA.

Resorting to the latter approach dealing with the unpredictable returning of primary services, in this paper, we study the spectrum harvesting and sharing problem for multi-hop CRNs under uncertain spectrum supply. To effectively harvest and share the under-utilized licensed spectrum, we introduce a new emerging service provider, called Secondary Service Provider (SSP). Suppose that the SSP has its own bands (i.e., basic bands) and is able to collectively harvest the available licensed bands. In order to facilitate the accessing of SUs without CR capability, we assume the SSP has already established some partial infrastructure with CR mesh routers ${ }^{1}$ at low cost to provide coverage in the area of interest. Those CR mesh routers have $\mathrm{CR}$ capability and are equipped with multiple $\mathrm{CR}$ radios. SUs can cooperate with CR routers for packet delivery. Under the guidance of the SSP, SUs access their nearby CR mesh routers using basic bands and deliver packets via $\mathrm{CR}$ mesh routers using both basic bands and harvested bands. In such a CRN, more spectrum reuse opportunities can be created and the network capacity can be increased.

As for spectrum sharing, we focus on the joint frequency scheduling and routing problem among CR mesh routers in multi-hop CRNs. Suppose that CR mesh routers collect traffic from end users/SUs and form a set of CR sessions, each of which is characterized by a pair of source and destination CR routers and has a certain rate requirement. The SSP may ask an interesting question: how much bandwidth is at least required to maintain these CR sessions considering the availability of spectrum resources at a certain confidence level w.r.t. all the constraints from multiple layers in CRNs. To put it in another way, in this paper, we are trying to address how the SSP performs OSA, frequency scheduling and multi-hop multi-path routing so that the required network-wide spectrum resource is minimized ${ }^{2}$, given the fact that the licensed spectrum supply cannot be guaranteed.

Inspired by the statistics of spectrum bands obtained on observation and experiments in [5], [7], [8], we novelly model the uncertain spectrum vacancy of a licensed band (i.e.,

\footnotetext{
${ }^{1}$ In the rest of this paper, we use the words CR router/CR mesh router/router interchangeably.

${ }^{2}$ We follow the same objective as that in [12], where the so-called spacebandwidth product defined in [13] is adopted as the performance metric in the setting of multi-hop CRNs.
}

available bandwidth for OSA) as a random variable satisfying certain distribution. This modeling explicitly distinguishes the joint routing and frequency scheduling problem in CRNs from that in single-channel single-radio networks [14], [15] or multi-channel multi-radio networks [16]-[19]. The reason is that in those networks, the bandwidth is always regarded as a constant value. Even compared with prior work in the literature of multi-hop CRNs [12], [20], the unique feature of uncertain spectrum supply makes the route selection and scheduling in our paper much more challenging as well ${ }^{3}$.

Within the proposed architecture of CRNs, we exploit a pair of $(\alpha, \beta)$ parameters to characterize the SSP's concerns about the CRNs and mathematically formulate the joint frequency scheduling and routing problem. Specifically, $\alpha$ denotes the targeted confidence level for the availability of the required network-wide spectrum resource, and $\beta$ denotes the targeted quality of CR communications. Besides, we demonstrate constraints from multiple layers under the situation that spectrum supply is uncertain. In particular, we pay special attention to modeling the unpredictable activities of primary services, scheduling and interference models, and multi-path routing constraints. We also dwell on how to integrate the bandwidth of different bands and calculate the sum of link capacity, when the vacant bandwidth of every licensed band is a random variable. We formulate an optimization problem with the objective of minimizing the required network-wide spectrum resource at an $(\alpha, \beta)$ level.

For a fixed pair of $(\alpha, \beta)$, the formulated optimization problem falls into a mixed integer non-linear programming and is proved to be NP-hard [21]. Aiming to derive a feasible solution, we present a sub-optimal algorithm for the NP-hard optimization. We first find a lower bound for the objective by relaxing the integer variables in scheduling and interference constraints. Then, we propose a coarse-grained fixing algorithm to iteratively determine binary integer variables exploiting a threshold, where the bandwidth integration and the sum of link capacity from different bands are computed using discrete Fourier transform (DFT) and inverse discrete Fourier transform (IDFT). As long as fixing all the integer variables, we can determine flow routing variables and solve the optimization problem. Since the solutions attained by the coarse-grained fixing algorithm is an upper bound for the optimization objective, we compare it with the lower bound we have developed earlier. Simulation results show that (i) the proposed coarse-grained fixing algorithm is near-optimal for any $(\alpha, \beta)$ level; (ii) compared with the expected bandwidth based solution, the $(\alpha, \beta)$ based one has better performance in the sense that it lowers down the blocking ratio of CR sessions and improves the spectrum utilization.

The rest of the paper is organized as follows. In Section II, we propose a novel architecture for spectrum harvesting and sharing, introduce the model of spectrum uncertainty, and present other related models in CRNs. In Section III, we mathematically describe scheduling and routing constraints in CRNs. In Section IV, We define bandwidth required at $\alpha$ level and formulate joint routing and scheduling as an NP-

\footnotetext{
${ }^{3}$ Although we have devoted some efforts to analyzing this problem in [1], there is a lack of a solid system architecture to support our theoretical study. Meanwhile, requirements of CR capability are imposed on SUs' handsets, since there is no cooperation between SUs and CR routers for packet delivery.
} 
hard optimization problem. We also find a lower bound for this optimization problem. In Section V, we develop a coarsegrained algorithm for a sub-optimal solution. After that, we conduct simulations and analyze the performance results in Section VI, and draw concluding remarks in Section VII.

\section{NETWORK MODEL}

\section{A. Spectrum Harvesting \& Opportunistic Spectrum Accessing}

We consider a novel multi-hop CRN consisting of the $\mathrm{SSP}$, a group of SUs, $\mathcal{N}=\{1,2, \cdots, n, \cdots, N\}$ CR mesh routers and a set of available licensed spectrum bands ${ }^{4}$ with unequal size of bandwidths as shown in Fig. 1. The SSP is an independent wireless service provider with its own spectrum, i.e., the SSP's basic bands (potentially congested already), and is able to collectively harvest the available licensed bands. The SSP has also deployed some CR mesh routers at low cost to facilitate the accessing of SUs. SUs are just end-users not subscribed to primary services. No specific requirements are imposed on the SUs' communication devices. They could be any devices using any accessing technologies (e.g. laptops or desktop computers using Wi-Fi, cell phones using GSM/GPRS, smart phones using 3G/4G/NxtG accessing technology, etc.). SUs can access the basic bands owned by the SSP, but they cannot be tuned to the harvested licensed frequency. The CR mesh routers deployed by the SSP have CR capability and are equipped with multiple CR radios.

Under the guidance of the SSP, SUs and CR mesh routers cooperate with each other for packet delivery. Specifically, the mobile SUs report their online traffic requests to their nearby $\mathrm{CR}$ mesh routers via basic bands. The fixed CR mesh routers collect traffic demands from different end-users, form unicast CR communication sessions, and deliver packets using both the leftover basic bands and harvested bands. For better network capacity, reuse of the SSP's basic spectrum and sharing of the harvested spectrum, the SSP coordinates those $\mathrm{CR}$ mesh routers and jointly conducts frequency scheduling and flow routing among them as shown in Fig. 1.

Suppose there are a set of $\mathcal{L}$ uni-cast communication sessions among these CR mesh routers. Let $s(l) / d(l)$ denote the source/destination $\mathrm{CR}$ router of session $l \in \mathcal{L}$, and $r(l)$ be the rate requirement of session $l$. Assume the SUs' usage of basic bands in the multi-hop CRNs is a priori information. The $\mathrm{CR}$ routers are able to use the rest of basic spectrum owned by the SSP. The CR routers are also allowed to communicate with each other by opportunistically accessing the licensed bands when the primary services are not active, but they must evacuate from these bands immediately when primary services become active. Considering the geographical location of the $\mathrm{CR}$ routers, the available spectrum bands at one $\mathrm{CR}$ router may be different from another one in the network. To put it in a mathematical way, let $\mathcal{M}=\{1,2, \cdots, m \cdots, M\}$ be the band set including the available basic bands and licensed bands for $\mathrm{CR}$ communications, and $\mathcal{M}_{i} \subseteq \mathcal{M}$ represent the set of available bands at $\mathrm{CR}$ mesh router $i \in \mathcal{N} . \mathcal{M}_{i}$ may be different from $\mathcal{M}_{j}$, where $j$ is not equal to $i$, and $j \in \mathcal{N}$, i.e., possibly $\mathcal{M}_{i} \neq \mathcal{M}_{j}$.

\footnotetext{
${ }^{4}$ Taking the least-utilized spectrum bands introduced in [12] for example we found that the bandwidth between $[1240,1300] \mathrm{MHz}$ (allocated to amateur radio) is $60 \mathrm{MHz}$, while bandwidth between [1525, 1710] $\mathrm{MHz}$ (allocated to mobile satellites, GPS systems, and meteorological applications) is $185 \mathrm{MHz}$.
}

\section{B. Modeling of Uncertain Spectrum Supply}

The unique feature of CRNs is the uncertain spectrum supply from licensed bands, or say, the unpredictable bandwidth occupancy of primary services. To model this key feature of CRNs, we make $W^{m}$ denote the unoccupied bandwidth of the available band $m \in \mathcal{M}$, where $W^{m}$ is a random variable considering the unpredictable activities of primary services. Note that for an available basic band, the unoccupied bandwidth is a constant and the transmissions of $\mathrm{CR}$ routers over this band are not affected by primary services because the basic band belongs to the SSP. However, in probability theory, a constant can be regarded as a special random variable that takes a constant value, regardless of any event that occurs [22], [23]. Therefore, the modeling of $W^{m}$ as a random variable is applicable not only to the available licensed bands but also to the available basic bands for CR communications.

It is common in the literature [24] that one may attempt to use $\boldsymbol{E}\left(W^{m}\right)$, the first order statistics of $W^{m}$ [5] to predict the white space as shown in Fig. 2. Although this measurement is intuitive and easy to quantify, it ignores so much significant information w.r.t. the activities of primary services that it may lead to the failure of traffic delivery between CR routers as depicted in the zoomed-in picture of Fig. 2. It should be noted that the statistical characteristics of $W^{m}$ contain abundant knowledge about the available bandwidth of band $m$ for CR routers' opportunistic accessing. For example, assume $W^{m}$ is normally distributed with $\boldsymbol{E}\left(W^{m}\right)=2$ and $\sigma_{W^{m}}=1$, i.e., $W^{m} \sim \mathcal{N}\left(2,1^{2}\right)$. Then, the probability that $W^{m} \leq 3$ is equal to $84.1 \%$.

\section{Other Related Models}

1) Transmission Range and Interference Range: Suppose all CR mesh routers use the same power for transmission, and the power spectral density from the transmitter is $Q$. A widely used model [12], [20], [25] for power propagation gain is

$$
g_{i j}=\gamma \cdot d_{i j}^{-n}
$$

where $n$ is the path loss factor, $\gamma$ is an antenna related constant, and $d_{i j}$ is the distance between CR routers $i$ and $j$. We assume that the data transmission is successful only if the received power spectral density at the receiver exceeds a threshold $Q_{T}$. Meanwhile, we assume interference becomes non-negligible only if it produces a power spectral density over a threshold of $Q_{I}$ at the receiver. Thus, the transmission range for a CR router is $R_{T}=\left(\gamma Q / Q_{T}\right)^{1 / n}$, which comes from $\gamma \cdot\left(R_{T}\right)^{-n} \cdot Q=Q_{T}$. Similarly, based on the interference threshold $Q_{I}\left(Q_{I}<Q_{T}\right)$, the interference range for a CR router is $R_{I}=\left(\gamma Q / Q_{I}\right)^{1 / n}$. It is obvious that $R_{I}>R_{T}$ since $Q_{I}<Q_{T}$.

2) Link Capacity: According to Shannon-Hartley theorem, if $\mathrm{CR}$ router $i$ sends data to $\mathrm{CR}$ router $j$ on link $(i, j)$ with band $m$, the capacity of link $(i, j)$ with band $m$ is $c_{i j}^{m}$ $=W^{m} \log _{2}\left(1+\frac{g_{i j} Q}{\eta}\right)$, where $\eta$ is the ambient Gaussian noise density. As we know, to mathematically model the link capacity is imperative in the sense that the aggregate flow rates on each radio link can never exceed this link's capacity, which is an important constraint for routing. Different from modeling of link capacity in the other wireless networks [15] or in existing literature [12], we are also aware that $c_{i j}^{m}$ is 


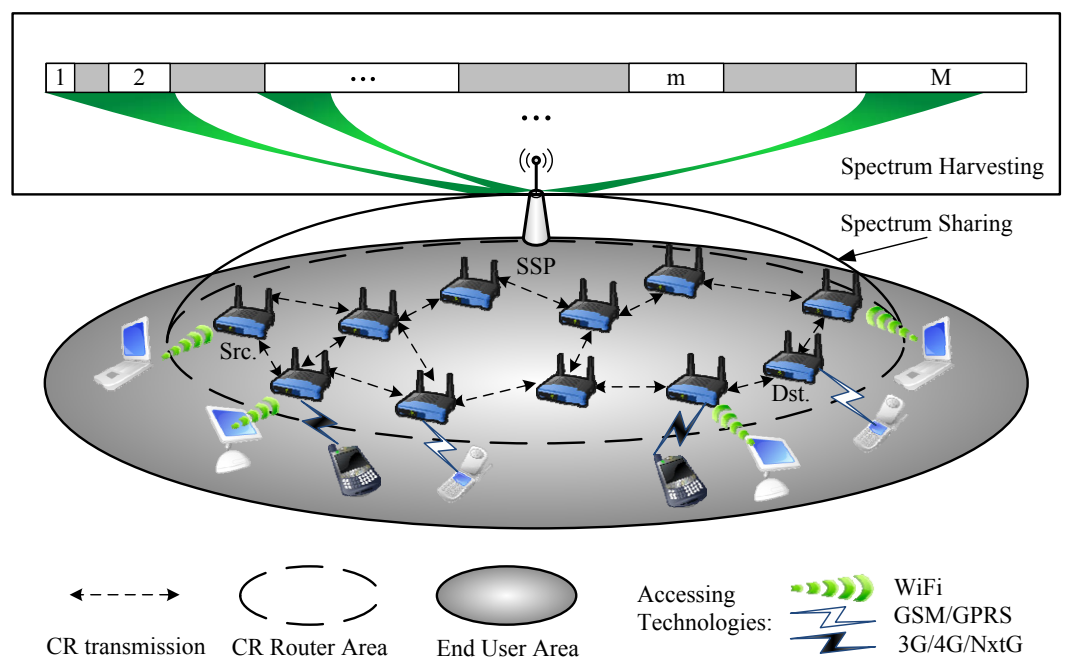

Fig. 1. A novel architecture for spectrum harvesting and sharing under uncertain spectrum supply in multi-hop CRNs.

not a fixed number but a random variable since the available bandwidth $W^{m}$ is uncertain in CRNs. Besides, note that the denominator inside the log function contains only $\eta$. This is because of one of our interference constraints, i.e., when CR router $i$ is transmitting to $\mathrm{CR}$ router $j$ on band $m$, then all the other neighbors of router $j$ within its interference range are prohibited from using this band. We will address the interference constraints in details in the following section.

\section{FREQUENCY SCHEDULING AND Routing CONSTRAINTS FOR OPPORTUNISTIC ACCESSING}

\section{A. Scheduling and Interference Constraints}

Scheduling can be conducted in time domain, in frequency domain, or in both of them. In this paper, we only focus on frequency based band assignment, i.e., how to assign bands at a CR mesh router for transmission and reception. A plausible scheduling on frequency bands must consider the limitations at the transmitter side and guarantee no interference at the receiver side.

Assume band $m$ is available at both router $i$ and router $j$, i.e., $m \in \mathcal{M}_{i} \cap \mathcal{M}_{j}$. We denote

$$
s_{i j}^{m}= \begin{cases}1 & \text { If router } i \text { transmits data to router } j \text { on } \\ & \text { band } m, \\ 0 & \text { otherwise. }\end{cases}
$$

For a router $i \in \mathcal{N}$ and a band $m \in \mathcal{M}_{i}$, denote $\mathcal{T}_{i}{ }^{m}$ the set of CR routers that can also opportunistically access band $m$ and are within the transmission range of router $i$, i.e.,

$$
\mathcal{T}_{i}^{m}=\left\{j: d_{i j} \leq R_{T}, j \neq i, m \in \mathcal{M}_{j}\right\} .
$$

From the view of the transmitter, CR router $i$ is not able to transmit to multiple routers on the same frequency band. Thus, we have

$$
\sum_{q \in \mathcal{T}_{i}^{m}} s_{i q}^{m} \leq 1
$$

From the view of the receiver, a CR router cannot use the same frequency band for transmission and reception ${ }^{5}$, due to "self-interference" at the physical layer. That is, if $s_{i j}^{m}=1$, then for any $q \in \mathcal{T}_{j}^{m}, s_{j q}^{m}$ must be 0 , i.e.,

$$
s_{i j}^{m}+\sum_{q \in \mathcal{T}_{j}^{m}} s_{j q}^{m} \leq 1 .
$$

Note that in (5), we are referring to a specific router $j$ to which router $i$ is transmitting. If $s_{i j}^{m}=1$, then $\sum_{q \in \mathcal{T}_{j}^{m}} s_{j q}^{m}=0$, i.e., $\mathrm{CR}$ router $j$ is not able to use the same frequency band $m$ for transmission. On the other hand, if $s_{i j}^{m}=0$, then $\sum_{q \in \mathcal{T}_{j}^{m}} s_{j q}^{m} \leq 1$, i.e., router $j$ may use band $m$ for transmission, but can only use it for one receiving router $q \in \mathcal{T}_{j}^{m}$, which is the same as in (4).

Beyond the constraints above at the receiver, there are also interference constraints from the other CR routers in CRNs. To be specific, for a frequency band $m$, if CR router $i$ uses this band for transmitting data to a CR router $j \in \mathcal{T}_{i}{ }^{m}$, then any other routers that may produce interference on $\mathrm{CR}$ router $j$ should not use this band ${ }^{6}$. To model this constraint, we let $\mathcal{P}_{j}^{m}$ represent the set of routers that can produce interference at $\mathrm{CR}$ router $j$ on band $m$, i.e.,

$$
\mathcal{P}_{j}^{m}=\left\{p: d_{p j} \leq R_{I}, p \neq j, \mathcal{T}_{p}^{m} \neq \emptyset\right\} .
$$

The physical interpretation of $\mathcal{T}_{p}^{m} \neq \emptyset$ in the above formula is that CR router $p$ may use band $m$ for a valid transmission to a CR router in $\mathcal{T}_{p}^{m}$ and then may cause interference to router $j$. Based on the definition of $\mathcal{P}_{j}^{m}$, we have

$$
s_{i j}^{m}+\sum_{q \in \mathcal{T}_{p}^{m}} s_{p q}^{m} \leq 1 \quad\left(p \in \mathcal{P}_{j}^{m}, p \neq i\right) .
$$

In (7), if $s_{i j}^{m}=1$, i.e., router $i$ uses band $m$ to transmit to router $j$, then any $\mathrm{CR}$ router $p$ that may interfere with $\mathrm{CR}$ router $j$ should not transmit on this band, i.e., $\sum_{q \in \mathcal{T}_{p}^{m}} s_{p q}^{m}=0$. Likewise, if $s_{i j}^{m}=0$, (7) reduces into (4), i.e., router $p$ may

\footnotetext{
${ }^{5}$ This limitation applies to both the transmitter and receiver. The reason to categorize it into the constraints of the receiver is for the ease of writing in the rest of paper. Also, as for this constraint, the roles of transmitter and receiver are symmetric and interchangeable.

6 "Hidden terminal" problem is a special case under this constraint.
} 


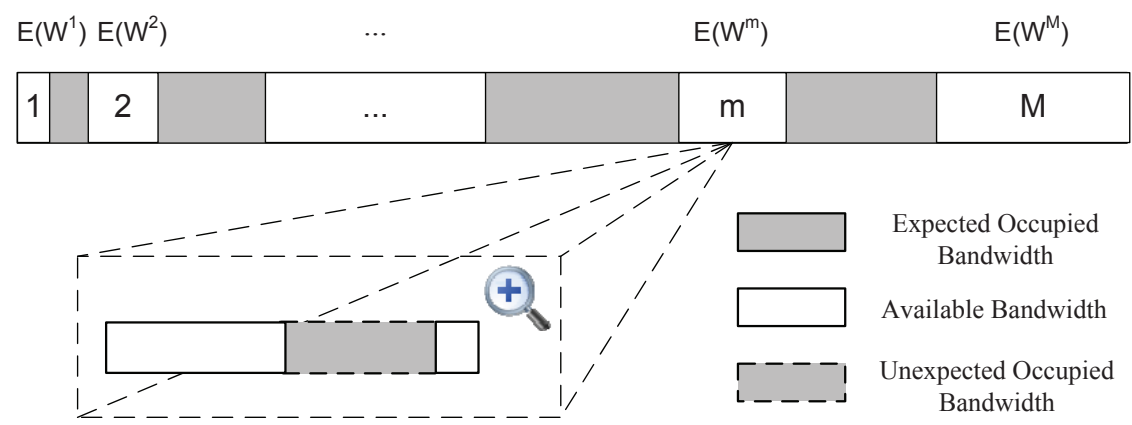

Fig. 2. A schematic illustrating available bandwidth for OSA and unpredictable occupation of primary services in CRNs.

transmit on band $m$ to one router $q \in \mathcal{T}_{p}^{m}$, i.e., $\sum_{q \in \mathcal{T}_{p}^{m}} s_{p q}^{m} \leq$ 1.

Now, we integrate the constraints in (5) and (7) into a general constraint at the receiver side. We define

$$
\mathcal{I}_{j}^{m}=\left\{p: d_{p j} \leq R_{I}, \mathcal{T}_{p}^{m} \neq \emptyset\right\}
$$

which is equivalent to

$$
\mathcal{I}_{j}^{m}= \begin{cases}\mathcal{P}_{j}^{m} \bigcup\{j\} & \text { If } \mathcal{T}_{j}^{m} \neq \emptyset \\ \mathcal{P}_{j}^{m} & \text { otherwise. }\end{cases}
$$

In this way, both (5) and (7) can be described by the following generalized constraint.

$$
s_{i j}^{m}+\sum_{q \in \mathcal{T}_{p}^{m}} s_{p q}^{m} \leq 1 \quad\left(p \in \mathcal{I}_{j}^{m}, p \neq i\right)
$$

\section{B. Routing Constraints}

As for routing, a source CR router may employ a number of relay CR routers to forward data packets toward its destination $\mathrm{CR}$ router. Obviously, there should be more than one path involved in data delivery since multi-path routing ${ }^{7}$ is more flexible to route the traffic from a source router to its destination. Following the routing model in [12], we mathematically present the constraints at network layer as follows.

Let $f_{i j}(l)$ denote the data rate on link $(i, j)$ that is attributed to session $l$, where $i \in \mathcal{N}, j \in \bigcup_{m \in \mathcal{M}_{i}} \mathcal{T}_{i}^{m}$, and $l \in \mathcal{L}$. To simplify the notation, let $\mathcal{T}_{i}=\bigcup_{m \in \mathcal{M}_{i}} \mathcal{T}_{i}^{m}$. then

If $\mathrm{CR}$ router $i$ is the source router of session $l$, i.e., $i=s(l)$,

$$
\sum_{j \in \mathcal{T}_{i}} f_{i j}(l)=r(l) .
$$

If CR router $i$ is an intermediate relay router for session $l$, i.e., $i \neq s(l)$ and $i \neq d(l)$, then

$$
\sum_{j \in \mathcal{T}_{i}}^{j \neq s(l)} f_{i j}(l)=\sum_{p \in \mathcal{T}_{i}}^{p \neq d(l)} f_{p i}(l) .
$$

If $\mathrm{CR}$ router $i$ is the destination router of session $l$, i.e., $i=d(l)$, then

$$
\sum_{p \in \mathcal{T}_{i}} f_{p i}(l)=r(l) .
$$

If (11) and (12) are satisfied, it can be easily verified that (13) must be satisfied. As a result, it is sufficient to

\footnotetext{
${ }^{7}$ The multiple radios of $\mathrm{CR}$ routers allow for multi-path routing.
}

list only (11) and (12) as routing constraints in the problem formulation.

In addition to the above flow balance equations at each router $i$ for each session $l$, the aggregate flow rates on each radio link cannot exceed this link's capacity. Taking interference constraints into consideration, the calculation of the link capacity $c_{i j}^{m}$ can be further simplified. When $s_{i j}^{m}=0$, we have $c_{i j}^{m}=0$. Thus, $c_{i j}^{m}$ should be written as

$$
c_{i j}^{m}=s_{i j}^{m} \cdot W^{m} \log _{2}\left(1+\frac{g_{i j} Q}{\eta}\right) .
$$

Therefore, for the requirement that the aggregate data rates on each link $(i, j)$ cannot exceed the link's capacity, we obtain

$$
\sum_{l \in \mathcal{L}}^{s(l) \neq j, d(l) \neq i} f_{i j}(l) \leq \sum_{m \in \mathcal{M}_{i} \cap \mathcal{M}_{j}} c_{i j}^{m} .
$$

\section{Problem Formulation ANd A LOWER Bound For THE CROSS-LAYER OPTIMIZATION}

The essential objective of CRNs is to avoid the waste of "white space" and to improve the spectrum utilization. Therefore, for the given number of CR routers' sessions with rate requirements, we seek for a feasible solution to assigning the available frequency bands to each router, scheduling bands for transmission and reception, and routing the flows so that the total radio bandwidth required in the multi-hop CRNs (so called space-bandwidth product [13]) is minimized.

Intuitively, the optimization problem can be formulated as follows [12].

\section{Min}

$$
\sum_{i \in \mathcal{N}} \sum_{m \in \mathcal{M}_{i}} \sum_{j \in \mathcal{T}_{i}^{m}} W^{m} s_{i j}^{m}
$$

$$
\text { s.t. } \quad(4),(10),(11),(12) \text { and (15), }
$$

where $s_{i j}^{m}$ and $f_{i j}(l)$ are optimization variables, and $g_{i j}, Q, \eta$ and $r(l)$ are all constants.

Note that due to the unpredictable returning of primary services, $W^{m}$ is not modeled as a constant but modeled as a random variable in CRNs as illustrated in Sec. II-C. This feature makes the spectrum resource minimization problem in this paper far different from that with guaranteed spectrum supply in existing works [12]. Therefore, two critical issues need to be addressed in the intuitive formulation above.

First, bandwidth integration in (16) is the sum of a series of random variables in CRNs rather than the sum of a series of deterministic quantities in other kinds of wireless networks. 
Second, with different choices of $s_{i j}^{m}$ and $f_{i j}(l)$, we have difficulty in comparing results of the optimization, i.e., $\sum_{i \in \mathcal{N}} \sum_{m \in \mathcal{M}_{i}} \sum_{j \in \mathcal{T}_{i}^{m}} W^{m} s_{i j}^{m}$, because they are random variables with different kinds of distribution.

\section{A. Problem Formulation}

1) Bandwidth Integration: We take a simple example to illustrate how to integrate the bandwidth of different bands. We let $W^{c}=W^{a}+W^{b}$, where $W^{a}$ and $W^{b}$ are independent, and bands $a, b \in \mathcal{M}$. Moreover, we assume the probability density function (PDF) and cumulative distribution function (CDF) of $W^{a}$ and $W^{b}$ are $h_{W^{a}}\left(w^{a}\right), h_{W^{b}}\left(w^{b}\right), H_{W^{a}}\left(w^{a}\right)$ and $H_{W^{b}}\left(w^{b}\right)$, respectively. Then, the CDF and PDF of $W^{c}$ are given below.

$$
\begin{aligned}
H_{W^{c}}\left(w^{c}\right) & =\int_{-\infty}^{\infty} \int_{-\infty}^{w^{c}-w^{a}} h_{W^{a}, W^{b}}\left(w^{a}, w^{b}\right) d w^{b} d w^{a} \\
& =\int_{-\infty}^{\infty} h_{W^{a}}\left(w^{a}\right) H_{W^{b}}\left(w^{c}-w^{a}\right) d w^{a} \\
h_{W^{c}}\left(w^{c}\right) & =\int_{-\infty}^{\infty} h_{W^{a}}\left(w^{a}\right) h_{W^{b}}\left(w^{c}-w^{a}\right) d w^{a} \\
& =\bigotimes_{m \in\{a, b\}} h_{W^{m}}\left(w^{m}\right),
\end{aligned}
$$

where $\otimes$ denotes the operator for the convolution of a sequence [22]. From the calculation of $h_{W^{c}}\left(w^{c}\right)$, we find that the sum of two independent random variables is associative and commutative in probability distribution. Using the same approach as in (17), this property can easily be extended to the sum of a finite number of independent random variables. For example, for the bandwidth integration of link $(i, j)$, the PDF of $W=\sum_{m \in \mathcal{M}_{i}} \cap \mathcal{M}_{j} W^{m} s_{i j}^{m}$ is

$$
h_{W}(w)=\bigotimes_{m \in \mathcal{M}_{i} \cap \mathcal{M}_{j}} h_{W^{m}}\left(w^{m}\right) s_{i j}^{m} .
$$

2) Bandwidth Required at $\alpha$ : Before we re-formulate the problem, we must quantify the bandwidth required for OSA when the vacancy of the licensed band is uncertain and modeled as a random variable. Thus, we leverage parameter $\alpha$ to define bandwidth required at $\alpha$ for OSA. Inspired by the mathematical expression of value at risk (VaR) in [26], we use $X_{\alpha}(w)$ to denote bandwidth required at $\alpha$ and define it as follows.

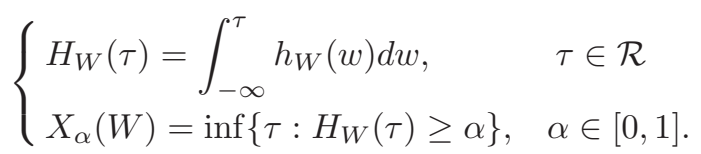

From (19), we find that the available bandwidth of the bandwidth integration for OSA is less than $X_{\alpha}(W)$ at confidence level $\alpha$ as shown in Fig. 3(a).

3) Formal Formulation: Based on the description of bandwidth integration and definition of bandwidth required at $\alpha$, the optimization problem can be reformulated as follows.

$$
\operatorname{Min} X_{\alpha}\left(\sum_{i \in \mathcal{N}} \sum_{m \in \mathcal{M}_{i}} \sum_{j \in \mathcal{T}_{i}^{m}} W^{m} s_{i j}^{m}\right)
$$

$$
\begin{gathered}
\text { s.t. } \sum_{q \in \mathcal{T}_{i}^{m}} s_{i q}^{m} \leq 1 \quad\left(i \in \mathcal{N}, m \in \mathcal{M}_{i}\right) \\
s_{i j}^{m}+\sum_{q \in \mathcal{T}_{p}^{m}} s_{p q}^{m} \leq 1 \quad\left(i \in \mathcal{N}, m \in \mathcal{M}_{i}, j \in \mathcal{T}_{i}^{m},\right. \\
\left.p \in \mathcal{I}_{j}^{m}, p \neq i\right) \\
\operatorname{Pr}\left(\sum_{l \in \mathcal{L}}^{s(l) \neq j, d(l) \neq i} f_{i j}(l) \leq \sum_{m \in \mathcal{M}_{i} \cap \mathcal{M}_{j}} W^{m} \log _{2}\left(1+\frac{g_{i j} Q}{\eta}\right) s_{i j}^{m}\right) \geq \beta \\
\sum_{j \in \mathcal{T}_{i}} f_{i j}(l)=r(l) \\
\sum_{j \neq s(l)} f_{i j}(l)-\sum_{p \in \mathcal{T}_{i}} f_{p i}(l)=0 \quad\left(l \in \mathcal{N}, j \in \mathcal{T}_{i}\right) \quad(22) \\
s_{i j}^{m}=0 \text { or } 1, f_{i j}(l) \geq 0 \\
(l \in \mathcal{L}, i \in \mathcal{L}, i \in \mathcal{N}, i \neq s(l)), d(l))
\end{gathered}
$$

Compared with the intuitive formulation, the reformulated problem mathematically solves the sum of random variables by using bandwidth integration and incorporates the other parameter $\beta$ to represent the SSP's requirements about quality of CR communications as presented in (22).

In addition, the objective of the optimization is clarified, i.e., to minimize bandwidth required at $\alpha$ to support the $\mathrm{CR}$ sessions with rate requirements, when joint scheduling and routing constraints are satisfied. Take $W$ and $W^{\prime}$ in Fig. 3(b) for example, assume they are both integrated bandwidths which satisfy all the constraints listed above. We can choose either $W$ or $W^{\prime}$ for OSA. We compare $X_{0.9}(W)$ and $X_{0.9}\left(W^{\prime}\right)$ as shown in Fig. 3(b), and decide to use $W$ for OSA. The reason is that if we choose $W$, the available bandwidth of bandwidth integration is less than $X_{0.9}(W)=9$ at confidence level of $90 \%$; but if we choose $W^{\prime}$, the available bandwidth of bandwidth integration is less than $X_{0.9}\left(W^{\prime}\right)=90$ at confidence level of $90 \%$. At an $(\alpha, \beta)$ level, the smaller $X_{\alpha}(W)$ is, the less spectrum required to maintain the set of $\mathrm{CR}$ sessions. The less spectrum required, the less CR sessions are affected by the activities of primary services.

Nevertheless, the above optimization problem itself is a mixed-integer nonlinear programming problem, which is proved to be NP-hard [21].

\section{B. The Lower Bound for the Cross-layer Optimization}

For an arbitrary pair of $(\alpha, \beta)$, the complexity of the problem formulated in Sec. IV-A arises from the binary $s_{i j}^{m}$ variables. To reduce the complexity and pursue a lower bound for the cross-layer optimization, we relax the binary requirement on $s_{i j}^{m}$ and replace it with $0 \leq s_{i j}^{m} \leq 1$. Due to the enlarged optimization space (caused by relaxation on $\left.s_{i j}^{m}\right)$, the solution to this relaxed optimization problem yields a lower bound for the minimization of bandwidth required at $\alpha$ problem in Sec. IV-A. Although the lower bound may not be achieved by a feasible solution, it offers a benchmark to measure the quality of feasible solutions.

\section{A Fast Fixing Algorithm for Sub-optimal SOLUTIONS USING DFT-IDFT}

Whereas we have the lower bound as the benchmark, we still seek for an effective and efficient solution to the proposed 


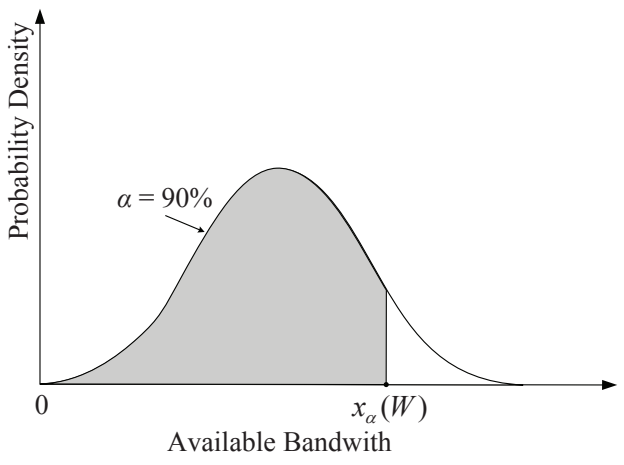

(a) The geometrical illustration of $X_{\alpha}(w)$.

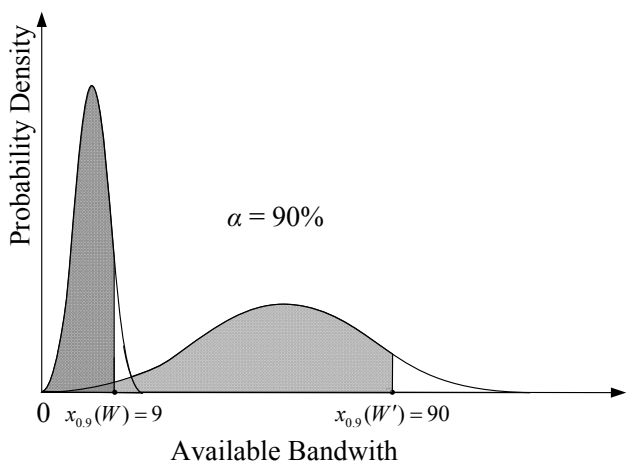

(b) The geometrical illustration of optimization objective.

Fig. 3. Bandwidth required at $\alpha$ and optimization objective.

problem since the $s_{i j}^{m}$ variables are binary values rather than real numbers within 0 and 1 . Given the values of the $(\alpha, \beta)$ pair, in this section, we first investigate how to reduce the complexity of computing the PDF convolution involved in the bandwidth integration. Then, with the knowledge of bandwidth integration computation, we present a coarse-grained fixing procedure to produce a feasible solution to the cross-layer optimization problem [12].

\section{A. Fast Computation of the Bandwidth Integration}

Following the typical way to efficiently calculate the linear convolution in [27], we implement the PDF convolution of bandwidth integration in Sec. IV-A in four steps.

Briefly speaking, we firstly convert the continuous PDF of $W^{m}$ into a discrete sequence by periodic sampling. Then, we zero-pad all the sequences, and compute the DFT of each sequence using the fast Fourier transform (FFT) algorithm (e.g., Cooley-Tukey algorithm). After that, we point-by-point multiply the DFTs of all the sequences, where the product represents the DFT of the PDF convolution of $W^{m}$. Finally, we compute the IDFT of the product, and convert the discrete result into continuous one to reconstruct the PDF convolution of the bandwidth integration.

\section{B. The Coarse-grained Fixing Procedure}

Now, the left problem is how to determine the $s_{i j}^{m}$ variables and fix flow routing in the problem formulation in Sec. IV-A. The key to simplifying the NP-hard optimization, fixing $f_{i j}(l)$ variables, and attaining an effective solution is the determination of the binary values for the $s_{i j}^{m}$ variables [12].
To determine the values of all the $s_{i j}^{m}$-variables, we iteratively solve a sequence of relaxed optimization problems. Considering interference constraints, in each iteration, we can fix at least one binary value for some $s_{i j}^{m}$. Specifically, for the first iteration, we relax all binary variables $s_{i j}^{m}$ to $0 \leq s_{i j}^{m} \leq 1$ as in Sec. IV-B to obtain a new optimization problem. We integrate the bandwidth with the PDF convolution and solve this new problem, so that we have a solution with each $s_{i j}^{m}$ being a value between 0 and 1 . Then, we select the $s_{i j}^{m}$ with the largest value among all the $s_{i j}^{m}$-values, and set this particular $s_{i j}^{m}$ to be 1 . In parallel with this fixing, by (20), we should set $s_{i q}^{m}=0$ for $\left(q \in \mathcal{T}_{i}^{m}, q \neq j\right)$. Meanwhile, by (21), we should set $s_{p q}^{m}$ to 0 for $\left(p \in \mathcal{I}_{j}^{m}, p \neq i, q \in \mathcal{T}_{p}^{m}\right)$. In particular, if the result includes more than one $s_{i j}^{m}$-variables with the value of 1 , we can set those $s_{i j}^{m}$-variables to 1 and perform an additional fixing for the largest fractional variable in the current iteration as illustrated above.

Having fixed some $s_{i j}^{m}$-variables in the first iteration, we remove all the terms associated with those already fixed $s_{i j}^{m}$ variables, eliminate the related constraints in (20) and (21), and update the problem to a new one for the second iteration. In the second iteration, we solve the new optimization and then determine the values of some other unfixed $s_{i j}^{m}$-variables based on the same process ${ }^{8}$. The iteration continues until we fix all $s_{i j}^{m}$-variables to be either 0 and 1 . The overall grained fixing procedure is summarized in Alg. 1.

Considering the number of bands with different frequencies and the spatial reuse in multi-hop CRNs, we may further reduce the complexity of the algorithm and speed up the procedure by fixing more $s_{i j}^{m}$-variables in a coarse-grained manner during each iteration. Firstly, the transmission in one band has no interference impact on the transmission in any other bands with different frequencies. Thus, for a link $(i, j)$, we may fix multiple bands within a single iteration in the coarse-grained fixing algorithm. Then, from the view of spatial reuse, a band can be used by the links far apart from one another (i.e., beyond the interference range of the routers in communications with a link). Thus, for a band $m$, we may fix multiple links that have no mutual interference within a single iteration in the coarse-grained fixing algorithm.

To be specific, we employ a threshold $\theta>0.5$ in the coarsegrained fixing process and fix all the $s_{i j}^{m}$-variables exceeding $\theta$ to 1 in a single iteration. To make sure that the constraints in (20) and (21) are held in the relaxed problem, we find that at most one variable $s_{i j}^{m}$ is allowed to be larger than $\theta$ in the local area in CRNs. Therefore, $\theta>0.5$ is suitable for determining the binary values of $s_{i j}^{m}$-values. In the case that none of the $s_{i j}^{m}$-variables exceed $\theta$, we will resort to the procedure listed in Alg. 1 for the current iteration and set the largest valued $s_{i j}^{m}$-variable to 1 .

Different from the lower bound obtained in Sec. IV-B, the proposed fast algorithm yields an upper bound to the problem formulated in Sec. IV-A. The quality of our suboptimal approach can be assessed by comparing its solution to the lower bound at various $(\alpha, \beta)$ levels.

\footnotetext{
${ }^{8}$ Provided that some $s_{i j}^{m}$-variables are fixed in the first iteration, the computation complexity in the second iteration is lower than that in the first iteration because we only need to deal with the remaining un-fixed $s_{i j}^{m}$ variables
} 


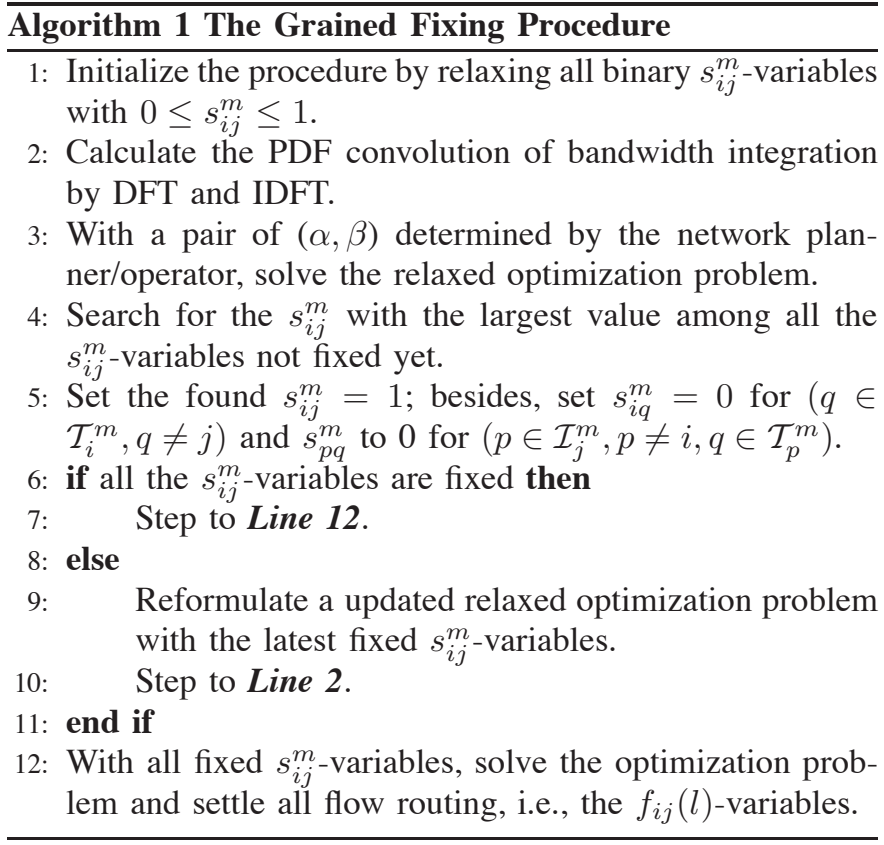

\section{Vi. Performance Analysis}

\section{A. Simulation Setup}

We conduct simulations with a CRN consisting of $|\mathcal{N}|=$ $25 \mathrm{CR}$ routers in a $50 * 50 \mathrm{~m}^{2}$ area. Among these CR routers, there are $|\mathcal{L}|=6$ active $\mathrm{CR}$ sessions, each session with a random rate requirement within $[10,100] \mathrm{Mb} / \mathrm{s}$. We assume the transmission range of each router to be $20 \mathrm{~m}$, the interference range to be $30 \mathrm{~m}$, the path loss index $n$ to be 4 and $\gamma$ to be 1 . For the simplicity of computation [12], we assume the threshold $Q_{T}$ is equal to the ambient Gaussian noise density, i.e., $\eta$. Thus, we have $Q_{I}=\left(\frac{20}{30}\right)^{n} Q_{T}$ and the transmission power spectral density $Q=(20)^{n} Q_{T} / \gamma=1.6 \cdot 10^{5} \eta$, according to the analysis in Sec. II-C. We also assume the basic bands of the SSP are fully utilized by the SUs without CR capability.

As for the uncertain spectrum supply, we assume that there are $|\mathcal{M}|=20$ licensed bands that can be opportunistically used by CR routers in the whole network. The vacant bandwidths of these bands are represented by a series of random variables. Based on data collected and the statistical analysis on spectrum utilization in [5], those random variables are exponentially distributed ${ }^{9}$, i.e., $h_{W^{m}}\left(w^{m}, \lambda^{m}\right)=\lambda^{m} e^{-\lambda^{m} w^{m}}$, where $\lambda^{m} \in(0,3]$. As we know, available bands for each CR router are a subset of these 20 bands based on its location, and the available bands for any two CR routers in the network may not be the same. Therefore, we randomly select a subset of bands from the spectrum pool of 20 bands for each router in the simulations. Due to different $\lambda^{m}$-values and random selection process, the size of available bandwidth in each band may be unequal, which truthfully mirrors the practical scenario.

It is not surprising that there may exist no feasible solution for some specific data set, because of dis-connectivity, inherent resource bottleneck in a hot spot, etc. In this paper, we only

\footnotetext{
${ }^{9}$ The results and analysis can easily be extended to other distributions (e.g., normal distribution, uniform distribution, etc.), even for the case that $W^{m}$ from different spectrum bands satisfies different distributions.
}

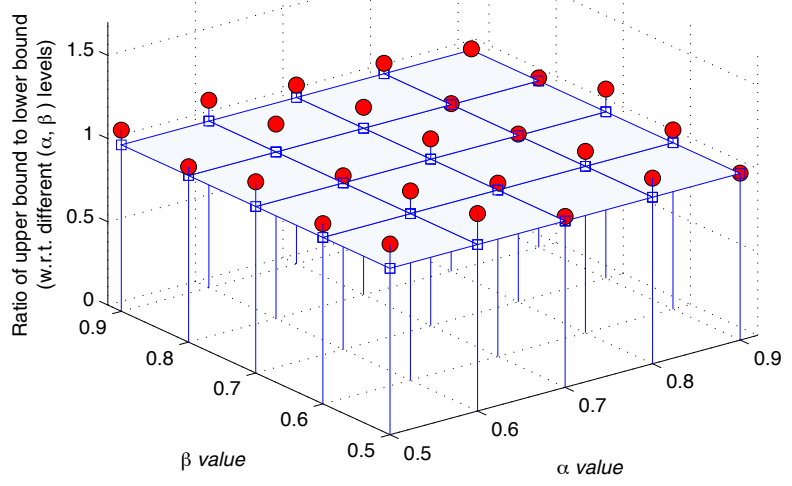

Fig. 4. Ratio of the upper bound to the lower bound at various $(\alpha, \beta)$ levels.

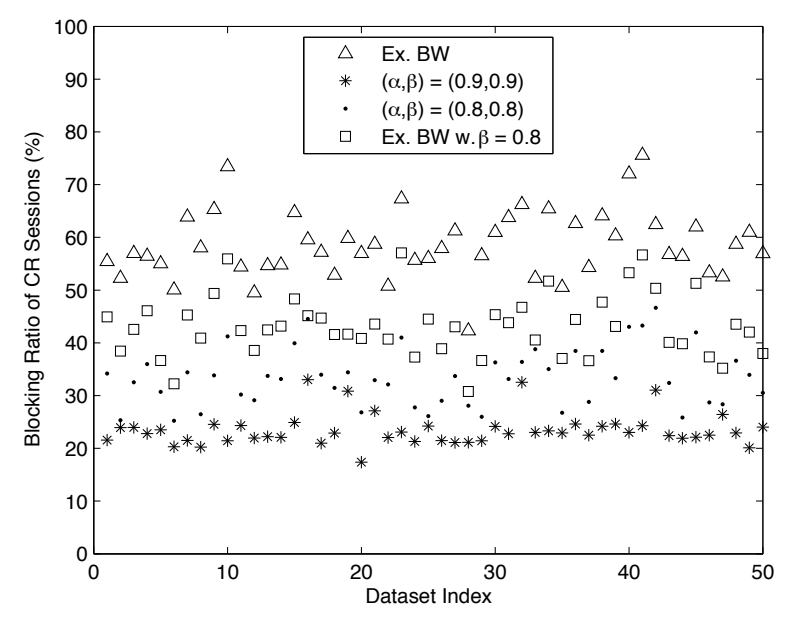

Fig. 5. The blocking ratio of different approaches.

focus on the data sets with feasible solutions and analyze the corresponding results as shown in the next subsection.

\section{B. Results and Analysis}

In Fig. 4, we evaluate the proposed coarse-grained fixing algorithm. We set $\theta=0.75$ (i.e., the threshold for the coarsegrained fixing), and compare the upper bound determined by the coarse-grained fixing algorithm with the lower bound developed in Sec. IV-B at different $(\alpha, \beta)$ levels. The range of $(\alpha, \beta)$ values is from $(50 \%, 50 \%)$ to $(90 \%, 90 \%)$, and simulations for the comparison of bounds are conducted for every $10 \%$ increase in either $\alpha$ or $\beta$ value. For each pair of $(\alpha, \beta)$, we employ 50 data sets that can produce feasible solutions and take the average value as a result. For each data set, we re-generate the network topology, source/destination pair and bit rate of each session, and available frequency bands for each CR router, which follows the guideline of simulation setup. As shown in Fig. 4 (the ratio is denoted by balls in shade; the benchmark of value 1 is denoted by the contour area intercepted and by the hollow squares at the sampled $(\alpha, \beta)$ pairs), the ratio of the upper bound to the lower bound via integer relaxation is equal to or slightly above to 1 in 
TABLE I

LOWER AND UPPER BOUNDS OF THE BANDWIDTH REQUIRED AT $\alpha$ FOR A GIVEN SET OF CR SESSIONS AT DIFFERENT $(\alpha, \beta)$ LEVELS.

\begin{tabular}{|c|c|c|c|c|}
\hline Index & $\alpha(\%)$ & $\beta(\%)$ & Lower-Bs & Upper-Bs \\
\hline 1 & 70 & 80 & 481.88 & 496.09 \\
\hline 2 & 75 & 80 & 517.29 & 517.29 \\
\hline 3 & 80 & 80 & 566.39 & 594.37 \\
\hline 4 & 85 & 80 & 628.71 & 631.15 \\
\hline 5 & 90 & 80 & 669.76 & 690.05 \\
\hline 6 & 70 & 85 & 490.28 & 493.25 \\
\hline 7 & 75 & 85 & 562.89 & 573.30 \\
\hline 8 & 80 & 85 & 589.02 & 595.13 \\
\hline 9 & 85 & 85 & 662.54 & 664.74 \\
\hline 10 & 90 & 85 & 714.15 & 725.87 \\
\hline 11 & 70 & 90 & 503.32 & 506.09 \\
\hline 12 & 75 & 90 & 602.34 & 606.50 \\
\hline 13 & 80 & 90 & 613.90 & 631.76 \\
\hline 14 & 85 & 90 & 681.88 & 696.09 \\
\hline 15 & 90 & 90 & 735.07 & 741.68 \\
\hline
\end{tabular}

almost all the area. The average ratio of the upper bound to the lower bound for all the sampled data sets is 1.0506, and the standard deviation is 0.0867 . It indicates that since the ratio of the upper bound to the lower bound is close to 1 at any $(\alpha, \beta)$ level, and the optimal bandwidth required at $\alpha$ is between those bounds, the solution found by the coarse-grained fixing algorithm must be close to the optimum.

Figure 5 shows the comparison between the proposed $(\alpha, \beta)$ based approach and the expected bandwidth based approach in which the expected value of bandwidth is used to characterize both the objective of the optimization and corresponding constraints [24]. For illustrative purposes, we compare the solution of the expected bandwidth based approach with the solutions obtained by the coarse-grained fixing algorithm at $(\alpha, \beta)=(80 \%, 80 \%),(\alpha, \beta)=(90 \%, 90 \%)$ and $\beta=80 \%$ with the expected value of required bandwidth as the objective, respectively. We take the blocking ratio of $\mathrm{CR}$ sessions as the evaluation metric and present simulation results for 50 data sets. From the results shown in Fig. 5, three observations can be made in order. First, the performance of the expected bandwidth based approach is worst of all because it ignores both the uncertainty of spectrum supply and the quality of CR communications when it selects the subset of licensed bands satisfying scheduling and routing constraints for OSA. The expected bandwidth with $\beta=80 \%$ approach is worse than the $(\alpha, \beta)$ based one because it also neglects the uncertain spectrum supply, i.e., the availability of required spectrum, as illustrated in Sec. IV-A3. By contrast, taking both factors into consideration, the $(\alpha, \beta)$ based approach performs the best. Second, for the $(\alpha, \beta)$ based approach, the blocking ratio decreases as the $(\alpha, \beta)$ level increases. Third, since the blocking ratio of $\mathrm{CR}$ sessions is closely associated with the spectrum utilization ratio in CRNs, i.e., low blocking ratio is equivalent to high spectrum utilization ratio for a given set of CR sessions. We can claim that the $(\alpha, \beta)$ based approach is better than the expected bandwidth based one in terms of spectrum utilization as well.

With a specific set of CR sessions (i.e., the network topology, the source/destination pair and the rate requirement of each session are fixed), Table I presents a general trend of change in terms of the bandwidth required at $\alpha$ in CRNs at different $(\alpha, \beta)$ levels. It is obvious that as $\alpha$-value increases, both the lower bound and the upper bound of the bandwidth required at $\alpha$ increase. Similarly, as $\beta$-value increases, both the bounds of the bandwidth required at $\alpha$ increase as well. The reason is from two aspects: i) From the optimization objective's point of view, the larger $\alpha$, the higher confidence level the network operator requests for the availability of required spectrum. The higher confidence level, the more bandwidth required at $\alpha$ is needed. ii) From the constraint's point of view, the larger $\beta$, the better quality of communications in CRNs. The better quality of CR communications, the more bandwidth required at $\alpha$ is needed.

\section{CONCLUSION}

In this paper, we have proposed a novel architecture of CRNs for spectrum harvesting and sharing, and presented a theoretical study on the joint frequency scheduling and routing problem in multi-hop CRNs under uncertain spectrum supply. We first introduce a new service provider, SSP, and let the SSP provide coverage in CRNs with low-cost CR routers in order to facilitate medium access for SUs without CR capability. Enlightened by the statistics of spectrum utilization, we then model the vacancy of an available band with a random variable satisfying certain statistical distribution. After that, we elaborate on scheduling and interference constraints as well as routing constraints with respect to the unpredictable activities of primary services. We also characterize the network performance with a pair of $(\alpha, \beta)$ parameters, and formulate an optimization problem to minimize the required network-wide spectrum resource at a $(\alpha, \beta)$ level for a set of CR sessions with rate requirements. To solve this NP-hard problem, we propose a coarse-grained fixing algorithm. We have demonstrated that the solution found by this algorithm is near-optimal and better than the expected bandwidth based solution in terms of blocking ratio and spectrum utilization.

\section{REFERENCES}

[1] M. Pan, C. Zhang, P. Li, and Y. Fang, "Joint routing and scheduling for cognitive radio networks under uncertain spectrum supply," in Proc. IEEE Conference on Computer Communications, INFOCOM 2011, Shanghai, China, April 2011.

[2] FCC, "Spectrum policy task force report," Report of Federal Communications Commission, Et docket No. 02-135, November 2002.

[3] J. Mitola, "Cognitive radio: An integrated agent architecture for software defined radio," Ph.D. Thesis, Royal Institute of Technology, Sweden, May 2000.

[4] I. Akyildiz, W. Lee, M. Vuran, and M. Shantidev, "Next generation/ dynamic spectrum access/ cognitive radio wireless networks: a survey," Computer Networks (Elsevier) Journal, vol. 50, no. 4, pp. 2127-2159, September 2006.

[5] D. Chen, S. Yin, Q. Zhang, M. Liu, and S. Li, "Mining spectrum usage data: a large-scale spectrum measurement study," in Proc. international conference on Mobile computing and networking, ACM Mobicom, 2009, Beijing, China, September 2009.

[6] S. M. Mishra, D. Cabric, C. Chang, D. Willkomm, B. V. Schewick, A. Wolisz, and R. W. Brodersen, "A real time cognitive radio testbed for physical and link layer experiments," in Proc. IEEE International Symposium on New Frontiers in Dynamic Spectrum Access Networks, DySPAN 2005, Baltimore, MD, November 2005.

[7] M. McHenry, "Spectrum white space measurements," New America Foundation Broadband Forum, June 20, 2003.

[8] M. A. McHenry, P. A. Tenhula, D. McCloskey, D. A. Roberson, and C. S. Hood, "Chicago spectrum occupancy measurements and analysis and a long-term studies proposal," in Proc. TAPAS 2006, Boston, MA, August 2006.

[9] J. H. Reed, Software Radio: A Modern Approach to Radio Engineering. New York: Prentice Hall, May 2002. 
[10] H. So, A. Tkachenko, and R. W. Brodersen, "A unified hardware/software runtime environment for FPGA based reconfigurable computers using borph," in Proc. International Conference on Hardware-Software Codesign and System Synthesis, Seoul, Korea, October 2006

[11] L. Duan, J. Huang, and B. Shou, "Cognitive mobile virtual network operator: Investment and pricing with supply uncertainty," in Proc. IEEE Conference on Computer Communications, INFOCOM 2010, San Diego, CA, March 2010 .

[12] Y. T. Hou, Y. Shi, and H. D. Sherali, "Spectrum sharing for multi-hop networking with cognitive radios," IEEE J. Sel. Areas Commun., vol. 26, no. 1 , pp. 146-155, January 2008

[13] X. Liu and W. Wang, "On the characteristics of spectrum-agile communication networks," in Proc. IEEE International Symposium on New Frontiers in Dynamic Spectrum Access Networks, DySPAN 2005, Baltimore, MD, November 2005.

[14] F. Chen, H. Zhai, and Y. Fang, "Available bandwidth in multirate and multihop wireless ad hoc networks," IEEE J. Sel. Areas Commun., vol. 28, no. 3, pp. 299-307, April 2010.

[15] X. Lin and N. B. Shroff, "The impact of imperfect scheduling on crosslayer congestion control in wireless networks," IEEE/ACM Trans. Netw., vol. 14, no. 2, pp. 302-315, April 2006.

[16] K. Jain, J. Padhye, V. N. Padmanabhan, and L. Qiu, "Impact of interference on multi-hop wireless network performance," in Proc. Mobile Computing and Networking, Mobicom '03, San Diego, CA, September 2003

[17] H. Li, Y. Cheng, C. Zhou, and P. Wan, "Multi-dimensional conflict graph based computing for optimal capacity in MR-MC wireless networks," in Proc. International Conference on Distributed Computing Systems, ICDCS 2010, Genoa, Italy, June 2010.

[18] M. Alicherry, R. Bhatia, and L. E. Li, "Joint channel assignment and routing for throughput optimization in multi-radio wireless mesh networks," in Proc. international conference on Mobile computing and networking, ACM Mobicom 2005, Cologne, Germany, August 2005.

[19] P. Kyasanur and N. H. Vaidya, "Capacity of multi-channel wireless networks: impact of number of channels and interfaces," in Proc. international conference on Mobile computing and networking, ACM Mobicom 2005, Cologne, Germany, August 2005.

[20] Z. Feng and Y. Yang, "Joint transport, routing and spectrum sharing optimization for wireless networks with frequency-agile radios," in Proc. IEEE Conference on Computer Communications, INFOCOM 2009, Rio de Janeiro, Brazil, April 2009.

[21] M. R. Garey and D. S. Johnson, Computers and Intractability: A Guide to the Theory of NP-Completeness. New York, NY: W. H. Freeman and Company, 1979.

[22] A. Papoulis and S. U. Pillai, Probability, Random Variables, and Stochastic Processes. McGraw-Hill, New York, 2002.

[23] W. Feller, An Introduction to Probability Theory and its Applications. New York, NY: John Wiley Sons Inc., 1971.

[24] M. Pan, Y. Song, P. Li, and Y. Fang, "Reward and risk for opportunistic spectrum accessing in cognitive radio networks," in Proc. IEEE Global telecommunications conference, Globecom 2009, Miami, FL, December 2010.

[25] A. Goldsmith, Wireless Communications. Cambridge, NY: Cambridge University Press, 2005.

[26] G. Holton, Value-at-Risk: Theory and Practice. Academic Press, 2003.

[27] A. V. Oppenheim and R. W. Schafer, Discrete-Time Signal Processing, 3rd ed. New York: Prentice Hall, 2009.

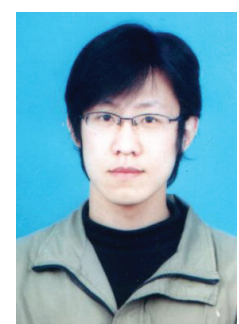

Miao Pan (S'07) received his B.E. degree in Electrical Engineering from Dalian University of Technology, China, in 2004 and M.E. degree in electrical and computer engineering from Beijing University of Posts and Telecommunications, China, in 2007. $\mathrm{He}$ has been working towards the Ph.D. degree in the Department of Electrical and Computer Engineering at University of Florida, Gainesville since August 2007. His research interests include cognitive radio communications and networking, cross-layer design and optimization, protocol design, and resource management in wireless networks and interdisciplinary systems.

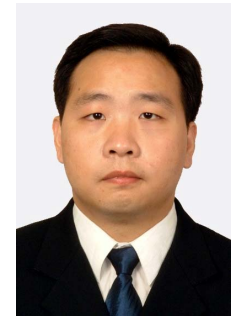

Chi Zhang (S'06-M'11) received his B.E. and M.E. degrees in Electrical and Information Engineering from Huazhong University of Science and Technology, Wuhan, China, in 1999 and 2002, respectively, and his Ph.D. degree in Electrical and Computer Engineering from the University of Florida, Gainesville, Florida, in 2011. In August 2011, he joined the School of Information Science and Technology at the University of Science and Technology of China, Hefei, China, where he is currently an Associate Professor. His research interests are in the areas of network protocol design, network performance analysis, and network security, particularly for wireless networks and social networks. Dr. Zhang is a member of IEEE.

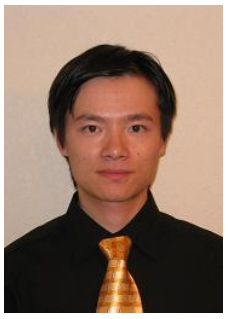

Pan Li (S'06-M'09) received the B.E. degree in electrical engineering from Huazhong University of Science and Technology, Wuhan, China, in 2005, and the Ph.D. degree in electrical and computer engineering from University of Florida, Gainesville, in 2009, respectively. He is currently an Assistant Professor in the Department of Electrical and Computer Engineering, Mississippi State University. His research interests include capacity and connectivity investigation, medium access control, routing algorithms, and cross-layer design and optimization in wireless networks. He is a member of the IEEE.

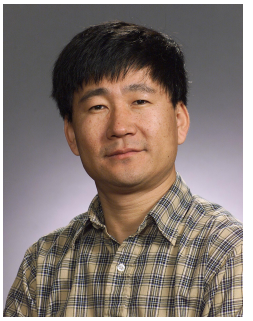

Yuguang "Michael" Fang (S'92-M'97-SM'99F'08) received a Ph.D. degree in Systems Engineering from Case Western Reserve University in January 1994 and a Ph.D degree in Electrical Engineering from Boston University in May 1997. He was an Assistant Professor in the Department of Electrical and Computer Engineering at New Jersey Institute of Technology from July 1998 to May 2000. $\mathrm{He}$ then joined the Department of Electrical and Computer Engineering at University of Florida in May 2000 as an Assistant Professor, got an early promotion to an Associate Professor with tenure in August 2003 and to a full professor in August 2005. He holds a University of Florida Research Foundation Professorship from 2006 to 2009, a Changjiang Scholar Chair Professorship with Xidian University, Xi'an, China, from 2008 to 2011, and a Guest Chair Professorship with Tsinghua University, China, from 2009 to 2012. He has published over 300 papers in refereed journals and conferences. Dr. Fang received the US National Science Foundation CAREER Award in 2001 and the Office of Naval Research Young Investigator Award in 2002, and is the recipient of the Best Paper Award in IEEE ICNP in 2006. He is currently serving as the Editor-in-Chief for IEEE Wireless Communications and serves/served on several editorial boards for several journals including IEEE Transactions on Communications, IEEE Transactions on Wireless Communications, and IEEE Transactions on Mobile Computing. $\mathrm{He}$ has been actively involved with IEEE INOFOCOM (TPC Co-Chair, 2014; TPC Vice-Chair, 2005, Area TPC Chair, 2009-2012; TPC member, 1998, 2000, 2003-2008). He is a Fellow of IEEE. 